\title{
COUNTING PATTERNS WITH A GIVEN AUTOMORPHISM GROUP
}

\author{
DENNIS E. WHITE ${ }^{1}$
}

\begin{abstract}
A formula, analogous to the classical Burnside lemma, is developed which counts orbit representatives from a set under a group action with a given stabilizer subgroup conjugate class. This formula is applied in a manner analogous to a proof of Pólya's theorem to obtain an enumeration of patterns with a given automorphism group.
\end{abstract}

1. Let $S$ be a finite set and $G$ a finite group acting on $S$. Let $\Delta$ be a system of orbit representatives for $G$ acting on $S$. The following theorem is well known:

Theorem 1 (Burnside [1]). For any function $\omega$ defined on $S$ satisfying $\omega(\sigma s)=\omega(s)$ for all $\sigma \in G$, for all $s \in S$, we have

$$
\sum_{s \in \Delta} \omega(s)=\frac{1}{|G|} \sum_{\sigma \in G} \sum_{s \in S} \omega(s) \chi(\sigma s=s)
$$

where

$$
\chi(\text { statement })= \begin{cases}1 & \text { if statement is true }, \\ 0 & \text { otherwise. }\end{cases}
$$

For $s \in S$ let $G_{s}=\{\sigma \in G: \sigma s=s\}$ be the stabilizer subgroup of $G$ at $s$. Let $G_{1}, G_{2}, \ldots, G_{N}$ be a complete set of nonconjugate subgroups of $G$, ordered such that $\left|G_{1}\right| \geq \cdots \geq\left|G_{N}\right|$. For any two subgroups $H, K \subset G$ we define

$$
M_{K}(H)=\frac{1}{|K|} \sum_{\sigma \in G} \chi\left(\sigma H \sigma^{-1} \subset K\right) .
$$

Received by the editors July 1, 1973.

AMS(MOS) subject classifications (1970). Primary 05A15, 20 B25; Secondary $05 \mathrm{C} 25$.

Key words and phrases. Orbit, stabilizer subgroup, conjugate subgroup, mark, pattern inventory, Möbius function.

${ }^{1}$ Research sponsored by the Air Force Office of Scientific Research, Air Force Systems Command, USAF, under AFOSR Contract/Grant No. 71-2089. 
$M_{K}(H)$ is sometimes called the mark of $K$ at $H$. The matrix $M=\left(M_{G_{j}}\left(G_{i}\right)\right)$ is triangular and $M_{G_{i}}\left(G_{i}\right) \geq 1$ so that we can define $B=M^{-1}, B=\left(b_{i j}\right)$. We also note that $M_{K}(H)$ is constant on conjugate subgroups of $G$.

In this paper we show the following result:

Theorem 2. For any function $\omega$ defined on $S$ satisfying $\omega(\sigma s)=\omega(s)$ for all $\sigma \in G$, for all $s \in S$, we have

$$
\sum_{s \in \Delta} \omega(s) \chi\left(G_{s} \sim G_{i}\right)=\sum_{j=1}^{N} b_{i j} \sum_{s \in S} \omega(s) \chi\left(G_{j} s=s\right),
$$

where $G_{s} \sim G_{i}$ means $G_{s}$ conjugate to $G_{i}$ and $G_{j} s=s$ means $s$ is fixed by all of $G_{j}$.

In an elegant paper [2], DeBruijn showed that Pólya's counting theorem [5] can be obtained from Theorem 1 upon letting $S=R^{D}$, where $R^{D}$ is the set of functions from the finite set $D=\{1,2, \ldots,|D|\}$ to the finite set $R=\{1,2, \ldots,|R|\}$, letting $G$ act on $D$ and hence on $R^{D}$ by setting $\sigma f(d)=$ $f\left(\sigma^{-1} d\right)$, and setting $\omega(f)=\Pi_{d \in D^{x} f(d)}$, where $x_{1}, x_{2}, \ldots$ are indeterminate. If we use the same approach, starting from Theorem 2 instead of Theorem 1 , with no additional difficulty we obtain a more refined version of Pólya's theorem.

Let $Q_{i}\left(x_{1}, x_{2}, \ldots\right)$ denote the pattern inventory for patterns whose automorphism group is conjugate to $G_{i}$ :

$$
Q_{i}\left(x_{1}, x_{2}, \cdots\right)=\sum_{f \in \Delta} \omega(f) \chi\left(G_{f} \sim G_{i}\right)
$$

Let $P_{i}\left(y_{1}, y_{2}, \ldots\right)$ denote the orbit index monomial:

$$
P_{i}\left(y_{1}, y_{2}, \cdots\right)=\prod_{d \in D} y_{d}^{q_{G} G_{i}(d)},
$$

where $q_{G_{i}}(d)=$ the number of orbits of $G_{i}$ acting on $D$ of length $d$, and $y_{1}, y_{2}, \ldots$ are indeterminates. Then we have

Theorem 3.

$$
Q_{i}\left(x_{1}, x_{2}, \cdots\right)=\sum_{j=1}^{N} b_{i j} P_{j}\left(y_{1}, y_{2}, \cdots\right)
$$

where we substitute $\Sigma_{r \in R} x_{r}^{i}$ for $y_{i}$.

This result was proved independently by Stockmeyer [8]. However, he obtained it only as a by-product of elaborate Möbius function techniques. 
We show here that Theorem 3 can be derived by simple algebraic manipulations.

We were led to this result by considering the general isomorph rejection problem in a multilinear setting [9], [10]. In this setting, besides Theorem 3, we have also derived from Theorem 2 a whole variety of results counting patterns with a given automorphism group. In particular, for example, we may let $G$ act on $R$ and $D$ or let $G$ act on $D$ and $H$ act on $R$. Or we may extend $S$ to be a cartesian product of finite function spaces, $G$ acting on each of them. Or we may observe that a theorem of Foulkes [3] is nothing more than Theorem 2 applied to a special function space.

2. We shall first prove Theorem 2. The weight function $\omega$ in this theorem is commonly thought of as a function from $S$ into an algebra, usually the algebra of polynomials.

Proof of Theorem 2. Note that for any subgroup $H \subset G, \Sigma_{\sigma \in G} \chi\left(\sigma H \sigma^{-1} \subset G_{s}\right)$ is constant on orbits of $S$, so if we denote the orbit of $s$ by $O_{s}$ and recall that $|G|=\left|G_{s}\right|\left|O_{s}\right|$ we have

$$
\begin{aligned}
\sum_{i=1}^{N} M_{G_{i}}(H) \sum_{s \in \Delta} \omega(s) \chi\left(G_{s} \sim G_{i}\right)=\sum_{s \in \Delta} \frac{\omega(s)}{\left|G_{s}\right|} \sum_{\tau \in G} \chi\left(\tau H \tau^{-1} \subset G_{s}\right) \\
=\sum_{s \in S} \frac{\omega(s)}{\left|G_{s}\right|\left|O_{s}\right|} \sum_{\tau \in G} \chi\left(\tau H \tau^{-1} \subset G_{s}\right) \\
=\frac{1}{|G|} \sum_{\tau \in G} \sum_{s \in S} \omega(\tau s) \chi\left(H \subset G_{\tau s}\right)=\sum_{s \in S} \omega(s) \chi\left(H \subset G_{s}\right) .
\end{aligned}
$$

Inverting $M$ gives our result.

We shall now use Theorem 2 to prove Theorem 3. The similarities between this proof and the proof of Pólya's theorem in [2] are obvious.

Proof of Theorem 3. Note that

$$
Q_{i}\left(x_{1}, x_{2}, \cdots\right)=\sum_{j=1}^{N} b_{i j} \sum_{f \in R^{D}} \omega(f) \chi\left(G_{j} f=f\right) .
$$

But $G_{j} f=f$ means $\sigma f=f$ for all $\sigma \in G_{j}$, or $f(d)=f\left(\sigma^{-1} d\right)$ for all $d \in D$, for all $\sigma \in G_{j}$. Thus, $f$ must be restricted to be constant on the orbits of $G_{j}$ acting on $D$. We can then define $f$ such that $G_{j} f=f$ by defining it on each orbit. Thus,

$$
\sum_{f \in R} \omega(f) \chi\left(G_{j} f=f\right)=\sum_{f \in R} \prod_{\operatorname{Orb}\left(G_{j}: D\right)} x_{f \in \operatorname{Orb}\left(G_{j}: D\right)}^{|A|}
$$


where $\operatorname{Orb}\left(G_{j}: D\right)$ is the set of orbits of $G_{j}$ acting on $D$. Using the familiar sum-product interchange gives

$$
\begin{aligned}
\sum_{f \in R^{D}} \omega(f) \chi\left(G_{j} f=f\right) & =\prod_{A \in \operatorname{Orb}\left(G_{j}: D\right)} \sum_{r \in R} x_{i}^{|A|} \\
& =\prod_{d \in D}\left(\sum_{r \in R} x_{r}^{d}\right)^{q} G_{j}^{(d)}=P_{j}\left(\sum_{r \in R} x_{r}, \sum_{r \in R} x_{r}^{2}, \cdots\right) \text {. Q.E.D. }
\end{aligned}
$$

\section{REFERENCES}

1. W. S. Burnside, Theory of groups of finite order, 2nd ed., Cambridge Univ. Press, Cambridge, 1911; Dover, New York, 1955. MR 16, 1086.

2. N. G. de Bruijn, "Pólya's theory of counting," in E. F. Beckenbach, Applied combinatorial mathematics, Wiley, New York, 1964. MR 30 \#4687.

3. H. O. Foulkes, On Redfield's range-correspondences, Canad. J. Math. 18 (1966), 1060-1071. MR 34 \#87.

4. A. Garsia, A presentation of the enumeration theory of Pólya and de Bruijn, Analysis seminar notes, University of California, San Diego, Calif., 1971.

5. G. Pólya, Kombinatorische Anzahlbestimmungen für Gruppen, Graphen und chemische Verbindungen, Acta Math. 68 (1937), 145-254.

6. J. H. Redfield, The theory of group-reduced distributions, Amer. J. Math. 49 (1927), 433-455.

7. J. Sheehan, The number of graphs with a given automorphism group, Canad. J. Math. 20 (1968), 1068-1076. MR 38 \#1031.

8. P. K. Stockmeyer, Enumeration of graphs with prescribed automorphism group, Ph. D. Thesis, University of Michigan, Ann Arbor, Mich., 1971.

9. D. E. White, Multilinear techniques in enumeration and list generation, $\mathrm{Ph} . \mathrm{D}$. Thesis, University of California, San Diego, Calif., 1973.

10. S. G. Williamson, Isomorph rejection and a theorem of de Bruijn, SIAM J . Comput. 2 (1973), 44-59.

DEPARTMENT OF MATHEMATICS, UNIVERSITY OF CALIFORNIA, SAN DIEGO, LA JOLLA, CALIFORNIA 92037

Current address: School of Mathematics, University of Minnesota, Minneapolis, Minnesota 55455 